\title{
Characterization of pre-Columbian
} (a) CrossMark artefacts in situ through handheld portable $X$-ray fluorescence spectrometry: the case of ceramics from the Mochica site of San José de Moro (Peru)

\author{
Nino Del-Solar-Velarde ${ }^{1 *}$, Stan Kinis ${ }^{2}$, Rémy Chapoulie ${ }^{1}$, Renaud Joannes-Boyau ${ }^{2}$ and Luis Jaime Castillo ${ }^{3}$
}

\begin{abstract}
:
The famous archaeological site of San José de Moro (SJM) located in the Jequetepeque Valley (JV) represents one of the best-preserved pre-Columbian Mochica cemeteries uncovered along the north coast of Peru. SJM was a regional ritual centre where elites and the general population of all $\mathrm{J}$ met to celebrate ceremonial events. Its role as a place of regional integration and coordination continued long after the disappearance of the Mochica and SJM was continuously occupied throughout the Late Mochica Period (8th-9th c. AD) and into the Transitional Period (9th-10th c. AD). Even though the abandonment of Mochica traditions in SJM (funerary practices and ceramic styles) appears quite rapid, SJM was constantly occupied whereby local traditions were being reshaped. During this time various styles of ritual ceramics from different traditions (Mochica, Cajamarca and Wari) were buried within ceremonial and funerary contexts. This research involves archaeometric studies of excavated painted diagnostic ceramic sherds representing four distinctive pre-Columbian typologies present at this site-Mochica fineline bichrome, Mochica fineline polychrome, coastal Cajamarca and Highland Cajamarca ceramics. For this study handheld portable X-ray fluorescence spectrometer (HHpXRF) instrumentation was operated in situ in order to (1) perform non-destructive trace element analysis (2) to assess if Cajamarca ceramics were further elaborated using the same raw materials employed by the Mochica potters of SJM, and (3) to establish whether or not Mochica and Cajamarca ceramics were truly technological markers in the lower part of the Jequetepeque valley.

Results: The chemical elements detected in the ceramic bodies by the portable technique and used for this analysis were: $\mathrm{K}, \mathrm{Ca}, \mathrm{Mn}, \mathrm{Fe}, \mathrm{Zn}, \mathrm{Ga}, \mathrm{As}, \mathrm{Rb}, \mathrm{Sr}$ and Y. All sherds that were identified as aesthetically different also differed chemically with the exception of the Mochica fineline bichrome and the Mochica fineline polychrome, which were produced in a coastal workshop using the same local raw materials. In addition, these sherds were not elaborated employing the same recipe used in the Coastal and Highland Cajamarca productions.

Conclusions: Mochica ceramic productions are stylistically and chemically different from both Coastal and Highland Cajamarca productions. Ceramics from San José de Moro are technological markers of two cultures (Mochica and Cajamarca) in the lower part of the Jequetepeque Valley that interacted during Late Mochica and through the Transitional periods.
\end{abstract}

\footnotetext{
*Correspondence: ninodelsolar@gmail.com

${ }^{1}$ Institut de Recherche sur les Archéomatériaux-Centre de Recherche en Physique Appliquée à I'Archéologie (IRAMAT-CRP2A) UMR 5060 CNRS, Université Bordeaux Montaigne, Bordeaux, France

Full list of author information is available at the end of the article
} 
Keywords: Mochica, Cajamarca, Ceramic, HHpXRF, San José de Moro, Peru

\section{Background}

The archaeological site of San José de Moro is one of the most remarkable pre-Columbian Mochica cemeteries in northern Peru and has been continuously excavated since 1991 by the Proyecto Arqueológico San José de Moro (PASJM) of the Pontificia Universidad Católica del Perú [1-5]. Tombs from this site have produced substantial discoveries of Mochica, Cajamarca and Wari ceramics in association with other artefacts (e.g. metals, semiprecious stones, textiles, etc.) within 'elite' and 'non-elite' funerary contexts throughout the Late Mochica Period (8th-9th c. AD) and the Transitional Period (9th-10th c. AD). The funerary evidence reveals a social scenario of intense inter-political interactions resulting in a sequence of events leading to catastrophic consequences [6].

Archaeological data from SJM suggests local and nonlocal ceramics represent social and ethnic markers. The local technical ceramic traditions buried inside the funerary contexts of the Late period and represented in particular by the famous Mochica fineline painted vessels were possibly subject to technological modifications due to the influence of numerous foreign highland ceramic traditions and technical styles (i.e. Cajamarca and Wari) in the region. Using this research this hypothesis is now being first tested using an archaeometric approach (HHpXRF in situ research on Mochica and Cajamarca ceramics is in its infancy and uncommon). Some authors analyzed Mochica fineline bichrome, Mochica fineline polychrome and Cajamarca vessels separately and employed the use of various techniques such as SEMEDS, cathodoluminescence, Raman spectrometry, X-ray diffraction and petrography [7-9].

This study involves the investigation of 56 ceramic fragments from four typologies commonly found for the Late Mochica and Transitional periods at SJM: (1) Mochica fineline bichrome (2) Mochica fineline polychrome (3) Coastal Cajamarca (4) Highland Cajamarca. The samples were excavated from three archaeological areas of SJM: area 47, area 49 and area 50 (Fig. 2). In area 47, layer 12 was excavated [10] and two Mochica polychrome fragments were identified. This deposit may be associated to the Late Mochica B occupation of the site (cf. [1,11] for archaeological time spans for San José de Moro site). In area 49 layer 5 was excavated [12] and temporally associated to the Late Mochica $\mathrm{B}$ or Late Mochica C occupations by a single Mochica polychrome fragment and two Cajamarca Cursivo Floral sherds located in this layer. Finally, in area 50, several Cajamarca Cursivo Floral fragments of layer 4 were revealed [13]. This layer may be associated to the late Transitional and even to the Early Lambayeque occupations of SJM.

It is important to note that while archaeometric research on Peruvian Pre-Columbian ceramics is advancing [7-9, 14-16], the use of HHpXRF technique in situ is very limited. This is the first time that archaeological Mochica and Cajamarca ceramics materials from the Mochica site of SJM have been analyzed using this technique. At present, only one other study using HHpXRF analysis on Cajamarca materials at Huancabamba Valley (15th-16th c. AD) has been completed [17]. Although in archaeology only $4 \%$ of publications cited using HHpXRF in an on-site-field-laboratory setting [18], it is ideally suited as an additional proxy for artefact processing at the archaeological site as a means to documenting large amounts of recovered objects (diagnostic ceramic sherds). On site field stations produce immediate feedback on the samples allowing the analyst instantaneous monitoring including any adjustments needed to the initial sampling strategy.

\section{The awkward adolescence of HHpXRF}

Even though the advent of HHpXRF instrumentation has caused considerable debate within the archaeology community, elemental analysis is now firmly in the ceramic archaeologist's lexicon, and even more now in 2016, HHpXRF systems have superior detector systems with the ability to accurately and precisely quantify the chemical composition of a range of ceramic materials incorporating the use of various factory calibrations and analytical protocols. The advent of HHpXRF should be seen as facilitating archaeological technology by eliminating the physical barriers between archaeology in the field and scientific analysis in the laboratory.

With the emergence of HHpXRF in recent years, numerous authors have questioned the quality control of chemical analyses in archaeology and see it to be declining, most notably accuracy and precision. Several researchers have raised strong concerns against the wholesale acceptance of HHpXRF data as "the be-all and end-all" answer to a broad range of archaeological questions instead of targeting it to more appropriate cases where quantitative data should only be published when analytical uncertainty can be addressed through measures of precision and accuracy [18, 19]. Killick believes HHpXRF used directly on inhomogeneous materials such as ceramics produces inaccurate and imprecise data, and should not be accepted for publication [20]. As 
a consequence, the HHpXRF based studies of ceramics presented at meetings and/or published are considered to poor science. Ultimately, the results from HHpXRF analyses must fulfill their own rules of validity and reliability, which is the baseline to good scientific research and this study. To answer these concerns of validation and sustainability, published research together with method protocols, raw data and other forms of supporting information is vital.

An extensive review (200 papers) of portable XRF (pXRF) systems and Handheld portable XRF (HHpXRF) instruments in recent archaeology demonstrate that only $43 \%$ of pXRF journals actually use handheld instruments, and of these, over $80 \%$ of HHpXRF analysis is performed in laboratory conditions (university laboratories and museums), with only $18 \%$ being conducted at an excavation (15\%) or on site in a field laboratory setting (3\%) [18].

Concerning the controversy over increased specialization and segmentation in archaeological practice [18, 21-25] the portability of HHpXRF has role to play that encourages bringing together scientific practice and archaeology. Investigation, analysis and interpretation need to be synchronized in the field and with common research objectives. This new approach to operating HHpXRF in the field is developing a new paradigm of new methods, theories and goals. The advent of the HHpXRF should be seen as facilitating archaeological technology by eliminating the physical barriers between archaeology in the field and scientific analysis in the laboratory.

This paper's focus is on pottery production at the preColumbian site of San José de Moro using this new noninvasive technique of chemical characterization. Two fundamental and central questions frame this research. The aim of this research is to answer both-Were Cajamarca ceramics elaborated further using the same raw materials employed by the Mochica potters of San José de Moro?-Were both Mochica fineline bichrome and polychrome painted ceramics produced using the same raw materials?

Although Mochica and Cajamarca vessels from SJM are well documented aesthetically [3-5], there is no comparative information about the nature of raw materials used to produce San José de Moro Mochica and Cajamarca ceramics at the Late Mochica and Transitional chronological periods. This technique (HHpXRF) has been chosen in order to compare the chemical composition of the samples without damaging them and using only raw intensity data.

\section{Methods}

\section{Samples}

56 pottery sherds $(\mathrm{n}=56)$ were chosen, attributed to and representative of four pre-Columbian typologies groups: Mochica fineline "bichrome" painted fragments $(\mathrm{n}=37)$, Mochica fineline "polychrome" painted fragments $(n=6)$, Coastal Cajamarca fragments $(n=7)$ and Highland Cajamarca fragments $(\mathrm{n}=6)$ (Figs. 1, 2).

\section{Characterization}

Now in 2016 many HHpXRF systems have superior detector resolution and electronics than laboratory-based systems of 5-10 years ago. Manufacturers have optimized their tube-sample-geometry; Bruker HHpXRF systems are configured with the tube and the detector at $64^{\circ}$ angle with sample testing at the convergence of this angle. In current XRF engineering other portable or fixed-laboratory XRF systems use angles that range from $45^{\circ}$ to $90^{\circ}$. With the miniaturization of electronic components, performance has vastly improved within the parameters of portable XRF systems. The manufacturers have created almost identical systems and repackaged the internal components (tube, detector, multichannel analyses) of portable bench-top systems into handheld configurations, the only difference being the packaging [19].

A Bruker Tracer III-SD Handheld X-ray Fluorescence Spectrometer was used in order to identify the chemical composition of the ceramic bodies in situ. The archaeological samples were lightly brushed and cleaned with water before non-invasive analysis was performed. The HHpXRF Spectrometer was positioned upward so that the sample table sat horizontally (the X-ray beam vertically upwards) with the protective cover above (sample shield). It is important to mention that we faced just one analytical constraint: the analyses were performed in a field laboratory where the environmental conditions (i.e. temperature, humidity, etc.) were not the same constantly during the campaign.

Regarding analytical parameters, analyses of heavier elements ranging from $\mathrm{K}$ to $\mathrm{Y}$ were performed whereby this methodology is favored and commonly used in archaeological ceramic research. The device uses a Rhodium target X-ray tube excitation source, a set of Palladium slits, $40 \mathrm{kV}$ high voltage and $30 \mu \mathrm{A}$ tube current. No vacuum was applied. A green filter composed of $\mathrm{Cu}$, $\mathrm{Ti}$ and $\mathrm{Al}$, was used for all analyses. Metered signals were collected from two to three separate unpainted sites from each sample (with the exception of BDX16316, BDX17166, BDX16340, BDX17164, BDX17165 and BDX16563 that had being analyzed just one time because of their complex geometries) over a period of 120 s. For each sample raw intensity data (the number of counts) were normalized to 1 and exported from S1XRF (Table 2). Because the nature of this data is compositional, intensity data has been transformed using the centred $\log$ ratio (CLR) in order to project it in Euclidean 

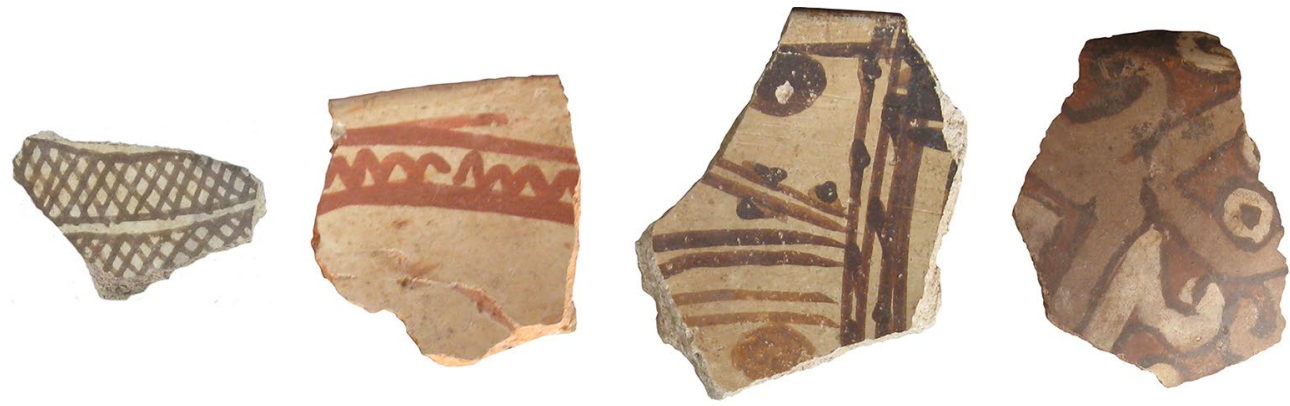

$2 \mathrm{~cm}$

Fig. 1 Mochica fineline "bichrome" painted fragment (BDX16319); Coastal Cajamarca fragment (BDX16553); Highland Cajamarca fragment (BDX16565) and Mochica fineline "polychrome" painted fragment (BDX16339)

space [26-28]. The CLR transformation is described in Eq. 1, where $g(x)$ is the geometric mean of a $D$ parts composition. Further statistical calculations were conducted using a fully cross-validated PCA using Past v2.17b Software Program [29].

$$
\operatorname{clr}(\mathrm{x})=[\ln (\mathrm{x} 1 / \mathrm{g}(\mathrm{x})), \ln (\mathrm{x} 2 / \mathrm{g}(\mathrm{x})), \ldots, \ln (\mathrm{xD} / \mathrm{g}(\mathrm{x}))]
$$

\section{Contextual analysis}

Contextual analysis is another important aspect in this methodology. The relationship of HHpXRF chemical analyses can add further structure to an understanding of the artefact in relation to its surroundings, and furthered with systematic studies of spatial patterning of the whole archaeological data. The concept of contact, exchange and dispersal is linked to regional scale distribution mapping; here HHpXRF methodology can construct and test theories such as central place theory and settlement hierarchies (particularly useful for regional chronologies in JV). The operation of the HHpXRF is new technique to distinguish geological sources and assign artefacts to them yielding valid archaeological (and not geochemical) data. Our results emphasize archaeological accuracy.

\section{Results}

Each sample was analyzed macroscopically in order to categorize the type of fragment (shape, body, form) and identified their aesthetic painted motifs and iconographies through comparison of handbook literature. For the classification of sherds handbook guides were employed-for Mochica ceramics, McClelland's information was primarily used [5] - for Coastal Cajamarca Bernuy and Bernal's classification was employed [30]and for Highland Cajamarca, data from Terada, Matsumoto and Watanabe allow classification of these materials [31-33]. The results are presented in Table 1.

The elements considered for HHpXRF analysis were: $\mathrm{K}$, $\mathrm{Ca}, \mathrm{Mn}, \mathrm{Fe}, \mathrm{Zn}, \mathrm{Ga}, \mathrm{As}, \mathrm{Rb}, \mathrm{Sr}$ and Y (Table 2). Primarily the study demonstrates Mochica fineline painted samples and Coastal Cajamarca samples have significantly higher $\mathrm{FeK} \alpha_{1}$ signals in contrast to the Highland Cajamarca samples that returned significantly higher $\mathrm{YK} \alpha_{1}$ signals. In addition, the Coastal Cajamarca samples returned a lower signal for $\mathrm{RbK} \alpha_{1}$ than that of the Mochica fineline painted styles while displaying similar $\mathrm{Sr}$ concentrations. Both Highland Cajamarca and Mochica fineline painted samples presented higher signals for $A s K \alpha_{1}$ than of the Coastal Cajamarca samples. All the samples have similar $\mathrm{ZnK} \alpha_{1}$ values.

Were Cajamarca ceramics elaborated further using the same raw materials used by the Mochica potters of San José de Moro? The PCA plot (Fig. 3) demonstrates that Mochica fineline painted samples, Coastal Cajamarca samples and Highland Cajamarca samples can be differentiated according to their respective chemical compositions. In our corpus, we differentiate three separate chemical groups being: Mochica productions (black dots), Coastal Cajamarca (gray diamonds) and Highland Cajamarca (gray triangles).

Were both Mochica fineline bichrome and polychrome painted ceramics produced using the same raw materials?

(See figure on next page.)

Fig. 2 Archaeological site and excavations at San José de Moro in 2013 and 2014. On the last image, area 47 is red, area 49 is green and area 50 is blue. Yellow area was also excavated, however no representative ceramics were found. Site location and archaeological areas showed using Google Earth images. (from Data SIO, NOAA, U.S. Navy, NGA, GEBCO; Images @ 2015 CNES/Astrium; Image @ 2015 DigitalGlobe; Google Earth) 

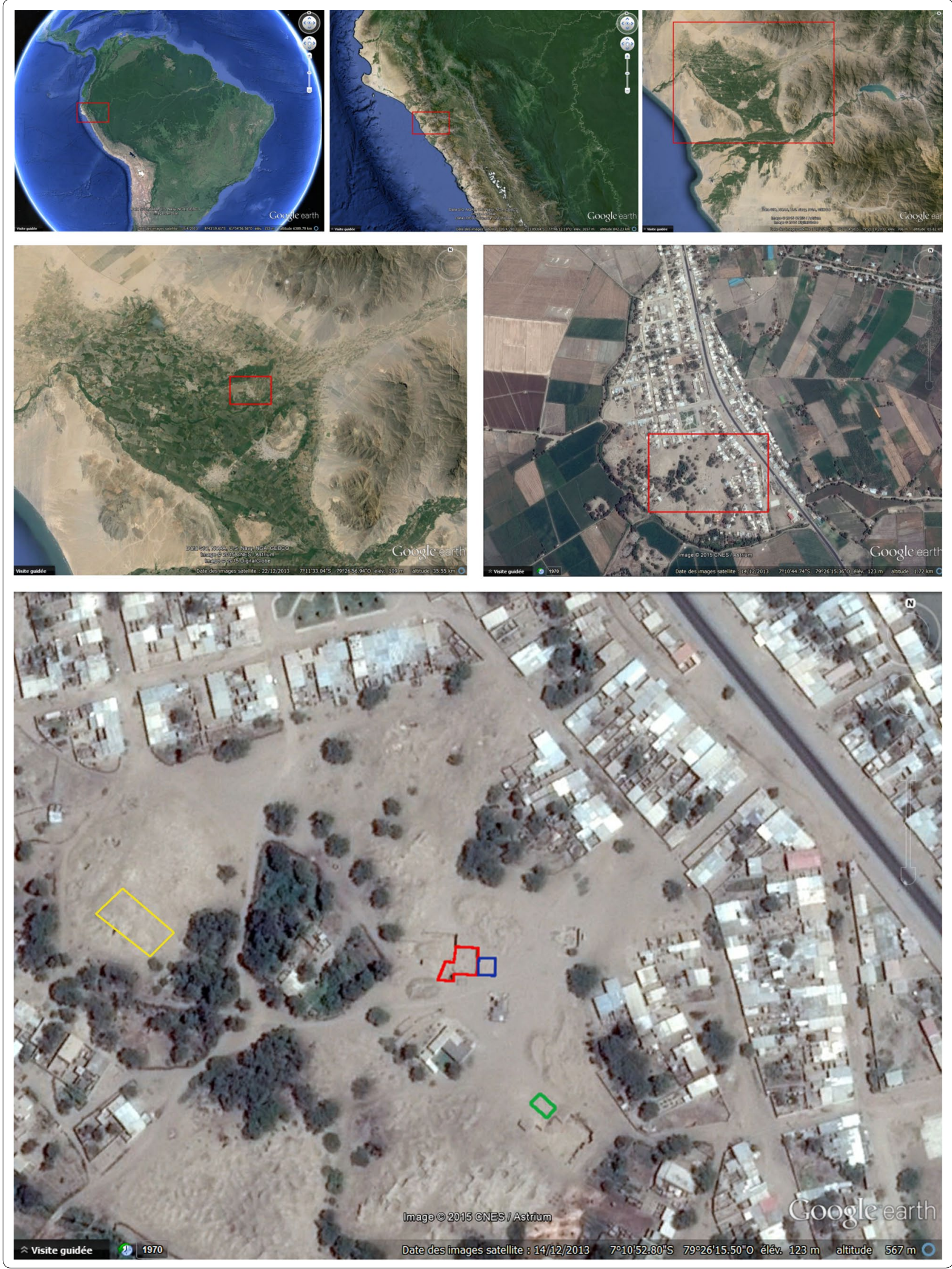
Table 1 Typological characterizations of pre-Columbian ceramics from San José de Moro

\begin{tabular}{|c|c|c|c|c|c|c|c|c|}
\hline No & Lab. name & $\begin{array}{l}\text { Archaeological } \\
\text { name }\end{array}$ & Fragment & Iconography & Reference & Type & Objet & Period \\
\hline 1 & BDX 16314 & A47-C12-FC15-01 & Body & Crescent boat & $\begin{array}{l}\text { McClelland } \\
\text { et al. [5] cf. } \\
\text { Figure 3.35, 3.42 }\end{array}$ & MFBB & Bottle or Jar & Late Mochica \\
\hline 2 & BDX 16315 & A47-C12-FC15-09 & Body & Reed boat & $\begin{array}{l}\text { McClelland } \\
\text { et al. [5] cf. } \\
\text { Figure } 3.12\end{array}$ & MFBB & Bottle or Jar & Late Mochica \\
\hline 3 & BDX 16316 & A47-C12-FC15-06 & Body & $\begin{array}{l}\text { Bean and stick } \\
\text { ceremony }\end{array}$ & $\begin{array}{l}\text { McClelland } \\
\text { et al. [5] cf. } \\
\text { Figure } 3.72\end{array}$ & MFBB & Bottle or Jar & Late Mochica \\
\hline 4 & BDX 16318 & A47-C12-FC26-05 & Body & $\begin{array}{r}\text { Bean and stick } \\
\text { ceremony (?) }\end{array}$ & $\begin{array}{l}\text { McClelland } \\
\text { et al. [5] cf. } \\
\text { Figure } 3.72\end{array}$ & MFBB & Bottle or Jar & Late Mochica \\
\hline 5 & BDX 16319 & $\begin{array}{l}\text { A47-C12- } \\
\quad \text { R254-FC10-01 }\end{array}$ & Body & Crescent boat & $\begin{array}{l}\text { McClelland } \\
\text { et al. [5] cf. } \\
\text { Figure 3.36, } 3.42\end{array}$ & MFBB & Bottle or Jar & Late Mochica \\
\hline 6 & BDX 16320 & A47-C12-FC24-03 & Body & Crescent boat & $\begin{array}{l}\text { McClelland } \\
\text { et al. [5] cf. } \\
\text { Figure 3.28, 3.31, } \\
3.42\end{array}$ & MFBB & Bottle or Jar & Late Mochica \\
\hline 7 & BDX 16321 & A47-C12-FC22-03 & Body & Lines & $\begin{array}{l}\text { McClelland et al. } \\
\text { [5] cf. Figure 3.7, } \\
\text { 3.169a }\end{array}$ & $\begin{array}{l}\text { MFBB or Mochica } \\
\quad V(?)\end{array}$ & Bottle or Jar & Late Mochica \\
\hline 8 & BDX 16322 & $\begin{array}{l}\text { A47-C12- } \\
\quad \text { R255-FC11-02 }\end{array}$ & Body & Crescent boat & $\begin{array}{l}\text { McClelland } \\
\text { et al. [5] cf. } \\
\text { Figure } 3.22,3.23 \\
3.42\end{array}$ & MFBB & $\begin{array}{l}\text { Bottle or Jar (body } \\
\text { with acute } \\
\text { angle) }\end{array}$ & Late Mochica \\
\hline 9 & BDX 16326 & A47-C12-FC24-02 & Body & Burial theme & $\begin{array}{l}\text { McClelland } \\
\text { et al. [5] cf. } \\
\text { Figure } 3.105\end{array}$ & MFBB & Bottle or Jar & Late Mochica \\
\hline 10 & BDX 16327 & A47-C12-FC29-01 & Body & $\begin{array}{l}\text { Birds with flying } \\
\text { fish in their } \\
\text { beaks (?) }\end{array}$ & $\begin{array}{l}\text { McClelland } \\
\text { et al. [5] cf. } \\
\text { Figure } 3.147\end{array}$ & MFBB & Bottle or Jar & Late Mochica \\
\hline 11 & BDX 16328 & A47-C12-FC24-01 & Body & $\begin{array}{l}\text { Muscovy duck } \\
\text { warrior (?), } \\
\text { Crested bird (?) }\end{array}$ & $\begin{array}{l}\text { McClelland } \\
\text { et al. [5] cf. } \\
\text { Figure } 3.155 a, \\
3.158\end{array}$ & MFBB & Bottle or Jar & Late Mochica \\
\hline 12 & BDX 16329 & A47-C12-FC26-06 & Body & Lines & $\begin{array}{l}\text { McClelland } \\
\text { et al. [5] cf. } \\
\text { Figure 3.169a }\end{array}$ & $\begin{array}{l}\text { MFBB or Mochica } \\
\quad V(?)\end{array}$ & Bottle or Jar & Late Mochica \\
\hline 13 & BDX 16330 & A47-C12-FC15-07 & Body & Lines & - & MFBB & Bottle or Jar & Late Mochica \\
\hline 14 & BDX 16331 & A47-C12-FC22-01 & Neck & Stripe & $\begin{array}{l}\text { McClelland et al. } \\
\text { [5] cf. Figure } 3.6\end{array}$ & MFBB & Bottle & Late Mochica \\
\hline 15 & BDX 16332 & $\begin{array}{l}\text { A47-C12- } \\
\text { R229-FC01-01 }\end{array}$ & Handle & Stripe & $\begin{array}{l}\text { McClelland } \\
\text { et al. [5] cf. } \\
\text { Figure 2.22b }\end{array}$ & MFBB & Bottle & Late Mochica \\
\hline 16 & BDX 16333 & A47-C12-FC26-04 & Body & Lines & - & MFBB & Bottle or Jar & Late Mochica \\
\hline 17 & BDX 16342 & A47-C12-FC26-07 & Handle & Stripe & $\begin{array}{l}\text { McClelland } \\
\text { et al. [5] cf. } \\
\text { Figure 2.22b }\end{array}$ & MFBB & Bottle & Late Mochica \\
\hline 18 & BDX 16343 & $\begin{array}{l}\text { A47-C12- } \\
\quad \text { R255-FC11-03 }\end{array}$ & Neck & Stripe & $\begin{array}{l}\text { McClelland } \\
\text { et al. [5] cf. } \\
\text { Figure } 3.10\end{array}$ & MFBB & Bottle & Late Mochica \\
\hline 19 & BDX 16345 & A47-C12-FC22-02 & Handle & $\begin{array}{l}\text { San José de Moro } \\
\text { Weapons }\end{array}$ & $\begin{array}{l}\text { McClelland et al. } \\
\text { [5] cf. Figure } 3.4\end{array}$ & MFBB & Bottle & Late Mochica \\
\hline 20 & BDX 17162 & $\begin{array}{l}\text { A49-RC5- } \\
\text { FC13b-01 }\end{array}$ & Handle & Stripe & $\begin{array}{l}\text { McClelland } \\
\text { et al. [5] cf. } \\
\text { Figure } 3.10\end{array}$ & MFBB & Bottle & Late Mochica \\
\hline
\end{tabular}


Table 1 continued

\begin{tabular}{|c|c|c|c|c|c|c|c|c|}
\hline No & Lab. name & $\begin{array}{l}\text { Archaeological } \\
\text { name }\end{array}$ & Fragment & Iconography & Reference & Type & Objet & Period \\
\hline 21 & BDX 17166 & A49-RC7-FC7-01 & Handle & Lines & $\begin{array}{l}\text { Donnan and } \\
\text { McClelland [4] } \\
\text { cf. Figure 5.70, } \\
5.73\end{array}$ & $\begin{array}{l}\text { MFBB or Mochica } \\
\text { IVN (?) }\end{array}$ & Bottle & Late Mochica \\
\hline 22 & BDX 17167 & A49-RC7-FC14-01 & Handle & Stripe & $\begin{array}{l}\text { McClelland } \\
\text { et al. [5] cf. } \\
\text { Figure } 3.10\end{array}$ & MFBB & Bottle & Late Mochica \\
\hline 23 & BDX 17168 & $\begin{array}{l}\text { A47-C13- } \\
\text { R283-FC12-01 }\end{array}$ & Body & Crescent boat & $\begin{array}{l}\text { McClelland } \\
\text { et al. [5] cf. } \\
\text { Figure 3.28, 3.31, } \\
3.42\end{array}$ & MFBB & Bottle or Jar & Late Mochica \\
\hline 24 & BDX 17169 & A47-C12-FC24-04 & Body & $\begin{array}{l}\text { Supernatural } \\
\text { confrontation: } \\
\text { Wrinkle Face } \\
\text { vs ... (?) }\end{array}$ & $\begin{array}{l}\text { McClelland } \\
\text { et al. [5] cf. } \\
\text { Figure } 3.50,3.51 \text {, } \\
\text { 3.55, } 3.67\end{array}$ & MFBB & Bottle or Jar & Late Mochica \\
\hline 25 & BDX 17170 & A47-C13-FC51-01 & Body & $\begin{array}{l}\text { Supernatural } \\
\text { confrontation }\end{array}$ & $\begin{array}{l}\text { McClelland } \\
\text { et al. [5] cf. } \\
\text { Figure } 3.49,3.58\end{array}$ & MFBB & Bottle or Jar & Late Mochica \\
\hline 26 & BDX 17171 & A47-C12-FC20-01 & Body & $\begin{array}{l}\text { Spiral and lines } \\
\text { (archaeological } \\
\text { re-painted) }\end{array}$ & $\begin{array}{l}\text { McClelland } \\
\text { et al. [5] cf. } \\
\text { Figure 2.10, 3.1, } \\
\text { 3.157, 3.144 }\end{array}$ & MFBB & Bottle or Jar & Late Mochica \\
\hline 27 & BDX 17172 & A49-C6-FC21-01 & Body & Burial Theme (?) & $\begin{array}{l}\text { McClelland } \\
\text { et al. [5] cf. } \\
\text { Figure } 3.111\end{array}$ & MFBB & Bottle or Jar & Late Mochica \\
\hline 28 & BDX 17173 & $\begin{array}{l}\text { A47-C13- } \\
\text { R296-FC39-03 }\end{array}$ & Body & $\begin{array}{l}\text { Anthropomor- } \\
\text { phized Crab (?), } \\
\text { Bean and stick } \\
\text { ceremony (?) }\end{array}$ & $\begin{array}{l}\text { McClelland et al. } \\
\text { [5] cf. } 3.129 \\
3.132\end{array}$ & $\begin{array}{l}\text { MFBB or Mochica } \\
\text { IVN (?) }\end{array}$ & Bottle or Jar & Late Mochica \\
\hline 29 & BDX 17174 & $\begin{array}{l}\text { A49-C8-Pa07- } \\
\text { FC22b-01 }\end{array}$ & Body & Lines & $\begin{array}{l}\text { McClelland } \\
\text { et al. [5] cf. } \\
\text { Figure 3.169a, } \\
\text { 3.169b }\end{array}$ & $\begin{array}{l}\text { MFBB or Mochica } \\
\quad V(?)\end{array}$ & Bottle or Jar & Late Mochica \\
\hline 30 & BDX 17175 & $\begin{array}{l}\text { A49-C9- } \\
\text { R141-FC16-01 }\end{array}$ & Body & $\begin{array}{l}\text { Geometric } \\
\text { designs }\end{array}$ & $\begin{array}{l}\text { McClelland } \\
\text { et al. [5] cf. } \\
\text { Figure } 3.169\end{array}$ & $\begin{array}{l}\text { MFBB or Mochica } \\
\quad \vee(?)\end{array}$ & Bottle or Jar & Late Mochica \\
\hline 31 & BDX 17176 & $\begin{array}{l}\text { A49-C6- } \\
\text { Pa3-FC12-01 }\end{array}$ & Body & Crested bird & $\begin{array}{l}\text { McClelland } \\
\text { et al. [5] cf. } \\
\text { Figure } 3.158\end{array}$ & MFBB & Bottle or Jar & Late Mochica \\
\hline 32 & BDX 17177 & A49-RC2-FC16-01 & Body & $\begin{array}{l}\text { Geometric } \\
\text { designs }\end{array}$ & $\begin{array}{l}\text { McClelland } \\
\text { et al. [5] cf. } \\
\text { Figure } 3.169\end{array}$ & MFBB & Bottle or Jar & Late Mochica \\
\hline 33 & BDX 17178 & A49-C8-FC03-01 & Body & Lines & $\begin{array}{l}\text { McClelland } \\
\text { et al. [5] cf. } \\
\text { Figure 3.169a, } \\
\text { 3.169b }\end{array}$ & MFBB & Bottle or Jar & Late Mochica \\
\hline 34 & BDX 17179 & $\begin{array}{l}\text { A49-C8- } \\
\text { R114-FC10-01 }\end{array}$ & Body & Crested bird & $\begin{array}{l}\text { McClelland } \\
\text { et al. [5] cf. } \\
\text { Figure } 3.158\end{array}$ & MFBB & Bottle or Jar & Late Mochica \\
\hline 35 & BDX 17180 & A49-C6-FC18-01 & Body & Reed boat & $\begin{array}{l}\text { McClelland et al. } \\
\text { [5] cf. Figure } 3.7\end{array}$ & MFBB & Bottle or Jar & Late Mochica \\
\hline 36 & BDX 17181 & A49-RC5-FC13-01 & Body & $\begin{array}{l}\text { Ceremonial } \\
\text { Badminton } \\
\text { scene (?) }\end{array}$ & $\begin{array}{l}\text { McClelland et al. } \\
\text { [5] cf. Figure } 392\end{array}$ & MFBB & Bottle or Jar & Late Mochica \\
\hline 37 & BDX 17182 & $\begin{array}{l}\text { A52- } \\
\text { RCD3a-FC10-01 }\end{array}$ & Body & Lines & - & MFBB & Bottle or Jar & Late Mochica (?) \\
\hline 38 & BDX 16334 & A47-C12-FC24-04 & Lip & Chevrons & $\begin{array}{l}\text { McClelland et al. } \\
\text { [5] cf. Figure 2.9, } \\
\text { 3.142, 3.145 }\end{array}$ & MFBP & Jar & Late Mochica \\
\hline
\end{tabular}


Table 1 continued

\begin{tabular}{|c|c|c|c|c|c|c|c|c|}
\hline No & Lab. name & $\begin{array}{l}\text { Archaeological } \\
\text { name }\end{array}$ & Fragment & Iconography & Reference & Type & Objet & Period \\
\hline 39 & BDX 16335 & $\begin{array}{l}\text { A47-C12- } \\
\text { R253-FC12-01 }\end{array}$ & Lip & Chevrons & $\begin{array}{l}\text { McClelland et al. } \\
\text { [5] cf. Figure 2.9, } \\
3.142,3.145\end{array}$ & MFBP & Jar & Late Mochica \\
\hline 40 & BDX 16339 & A49-RC5-FC05-01 & Body & Diamond & $\begin{array}{l}\text { McClelland } \\
\text { et al. [5] cf. } \\
\text { Figure } 3.175\end{array}$ & MFBP & Bottle & Late Mochica \\
\hline 41 & BDX 16340 & A49-RC4-FC03-01 & Body & Volute & $\begin{array}{l}\text { McClelland } \\
\text { et al. [5] cf. } \\
\text { Figure } 3.176\end{array}$ & MFBP & $\begin{array}{l}\text { Bottle (body with } \\
\text { acute angle) }\end{array}$ & Late Mochica \\
\hline 42 & BDX 17164 & $\begin{array}{l}\text { A49-C6- } \\
\text { R78-FC09-01 }\end{array}$ & Handle & $\begin{array}{l}\text { San José de Moro } \\
\text { Weapons }\end{array}$ & $\begin{array}{l}\text { McClelland et al. } \\
\text { [5] cf. Figure } 3.4\end{array}$ & MFBP & Bottle & Late Mochica \\
\hline 43 & BDX 17165 & $\begin{array}{l}\text { A49-C8- } \\
\text { Pa07-FC22-01 }\end{array}$ & Handle & $\begin{array}{l}\text { San José de Moro } \\
\text { Weapons }\end{array}$ & $\begin{array}{l}\text { McClelland et al. } \\
\text { [5] cf. Figure } 3.4\end{array}$ & MFBP & Bottle & Late Mochica \\
\hline 44 & BDX 16553 & A47-C12-FC15-02 & Lip & Type II & $\begin{array}{l}\text { Bernuy and Bernal } \\
{[30]}\end{array}$ & $\mathrm{CC}$ & Plate & Late Mochica \\
\hline 45 & BDX 16554 & $\begin{array}{l}\text { A47-Ext- } \\
\text { N2-FC04-02 }\end{array}$ & Lip & Type IV & $\begin{array}{l}\text { Bernuy and Bernal } \\
\text { [30] }\end{array}$ & CC & Plate & Transitional (?) \\
\hline 46 & BDX 16555 & A50-RC4-FC32-01 & Lip & Type IV & $\begin{array}{l}\text { Bernuy and Bernal } \\
\text { [30] }\end{array}$ & $\mathrm{CC}$ & Plate & $\begin{array}{l}\text { Transitional - } \\
\text { Lambayeque }\end{array}$ \\
\hline 47 & BDX 16556 & $\begin{array}{l}\text { A50-RC4- } \\
\text { FC36a-01 }\end{array}$ & Lip & Type IV & $\begin{array}{l}\text { Bernuy and Bernal } \\
\text { [30] }\end{array}$ & CC & Plate & $\begin{array}{l}\text { Transitional - } \\
\text { Lambayeque }\end{array}$ \\
\hline 48 & BDX 16557 & A50-RC4-FC38-01 & Body & Type IV & $\begin{array}{l}\text { Bernuy and Bernal } \\
\text { [30] }\end{array}$ & $C C$ & Plate & $\begin{array}{l}\text { Transitional - } \\
\text { Lambayeque }\end{array}$ \\
\hline 49 & BDX 16558 & $\begin{array}{l}\text { A50-RC4- } \\
\text { NO-FC30-01 }\end{array}$ & Body & Type IV & $\begin{array}{l}\text { Bernuy and Bernal } \\
{[30]}\end{array}$ & CC & Plate & $\begin{array}{l}\text { Transitional - } \\
\text { Lambayeque }\end{array}$ \\
\hline 50 & BDX 16561 & $\begin{array}{l}\text { A50-RC4- } \\
\text { NO-FC30-02 }\end{array}$ & Body & Type IV & $\begin{array}{l}\text { Bernuy and Bernal } \\
\text { [30] }\end{array}$ & CC & Plate & $\begin{array}{l}\text { Transitional - } \\
\text { Lambayeque }\end{array}$ \\
\hline 51 & BDX 16559 & $\begin{array}{l}\text { A50-RC4- } \\
\text { NO-FC30-03 }\end{array}$ & Lip & Cursivo Floral & $\begin{array}{l}\text { Terada and } \\
\text { Matsumoto } \\
{[31], \text { Watanabe }} \\
{[32,33]}\end{array}$ & $\mathrm{HC}$ & Plate & $\begin{array}{l}\text { Transitional - } \\
\text { Lambayeque }\end{array}$ \\
\hline 52 & BDX 16563 & A50-RC4-FC32-02 & Lip & Cursivo Floral & $\begin{array}{l}\text { Terada and } \\
\text { Matsumoto } \\
{[31], \text { Watanabe }} \\
{[32,33]}\end{array}$ & $\mathrm{HC}$ & Plate & $\begin{array}{l}\text { Transitional - } \\
\text { Lambayeque }\end{array}$ \\
\hline 53 & BDX 16564 & A50-RC4-FC32-03 & Foot & Cursivo Floral & $\begin{array}{l}\text { Terada and } \\
\text { Matsumoto } \\
{[31], \text { Watanabe }} \\
{[32,33]}\end{array}$ & $\mathrm{HC}$ & Plate & $\begin{array}{l}\text { Transitional - } \\
\text { Lambayeque }\end{array}$ \\
\hline 54 & BDX 16565 & A50-RC4-FC36-04 & Lip & Cursivo Floral & $\begin{array}{l}\text { Terada and } \\
\text { Matsumoto } \\
{[31] \text {, Watanabe }} \\
{[32,33]}\end{array}$ & $\mathrm{HC}$ & Plate & $\begin{array}{l}\text { Transitional - } \\
\text { Lambayeque }\end{array}$ \\
\hline 55 & BDX 16566 & $\begin{array}{l}\text { A49-C5- } \\
\text { Pa05-FC07-01 }\end{array}$ & Body & Cursivo Floral & $\begin{array}{l}\text { Terada and } \\
\text { Matsumoto } \\
{[31], \text { Watanabe }} \\
{[32,33]}\end{array}$ & $\mathrm{HC}$ & Plate & Late Mochica \\
\hline 56 & BDX 16567 & A49-RC5-FC10-01 & Lip & Cursivo Floral & $\begin{array}{l}\text { Terada and } \\
\text { Matsumoto } \\
{[31], \text { Watanabe }} \\
{[32,33]}\end{array}$ & $\mathrm{HC}$ & Plate & Late Mochica \\
\hline
\end{tabular}

N.b. MFBB Mochica fineline bichrome painted sherd of San José de Moro, MFBP Mochica fineline polychrome painted sherd of San José de Moro, CC Coastal Cajamarca, HC Highland Cajamarca

Since Mochica fragments are the most abundant, a comparative analysis of ratios was the first approach employed for comparison. Two chemical sub-groups identified as SJM-1 and SJM-2 were principally detected. In the SJM-1 sub-group fragments showed a $\mathrm{SrK} \alpha_{1} / \mathrm{FeK}_{1}$ ratio of $0.12-0.25$ and a $\mathrm{RbK \alpha} \alpha_{1} / \mathrm{SrK \alpha}_{1}$ ratio of $0.15-0.38$. 
Table $2 \mathrm{Ka}_{1}$ intensities on body ceramics obtained by HHpXRF analysis

\begin{tabular}{|c|c|c|c|c|c|c|c|c|c|c|c|}
\hline & Sample ID & $\mathrm{KKa}_{1}$ & $\mathrm{CaKa}_{1}$ & $\mathrm{MnKa}_{1}$ & $\mathrm{FeKa}_{1}$ & $\mathrm{ZnKa}_{1}$ & $\mathrm{GaKa}_{1}$ & $\mathrm{AsKa}_{1}$ & $\mathrm{RbKa}_{1}$ & $\mathrm{SrKa}_{1}$ & $\mathrm{YKa}_{1}$ \\
\hline \multirow[t]{2}{*}{1} & BDX16314 & 2.3 & 3.3 & 1.6 & 74.7 & 1.2 & 0.5 & 1.6 & 3.3 & 9.6 & 1.9 \\
\hline & $\sigma$ & 0.2 & 0.1 & 0 & 0 & 0 & 0 & 0.3 & 0 & 0.1 & 0.2 \\
\hline \multirow[t]{2}{*}{2} & BDX16315 & 2.4 & 3.9 & 1.6 & 73.3 & 1.1 & 0.4 & 0.8 & 2.7 & 12 & 1.8 \\
\hline & $\sigma$ & 0.2 & 0.5 & 0.1 & 0.1 & 0 & 0 & 0 & 0.1 & 0.6 & 0.1 \\
\hline 3 & BDX16316 & 2.3 & 4.6 & 1.6 & 74.2 & 1.3 & 0.4 & 0.8 & 3 & 10 & 1.9 \\
\hline \multirow[t]{2}{*}{4} & BDX16318 & 2.4 & 5.8 & 1.5 & 65.4 & 1.3 & 0.6 & 0.8 & 3.3 & 16.8 & 2.1 \\
\hline & $\sigma$ & 0.1 & 0.2 & 0 & 1 & 0 & 0 & 0 & 0.2 & 0.8 & 0.2 \\
\hline \multirow[t]{2}{*}{5} & BDX16319 & 2.7 & 5.2 & 1.7 & 70.6 & 1.2 & 0.5 & 0.7 & 2.4 & 13.1 & 1.8 \\
\hline & $\sigma$ & 0.1 & 0.5 & 0 & 0.2 & 0 & 0 & 0 & 0.1 & 0.4 & 0 \\
\hline \multirow[t]{2}{*}{6} & BDX16320 & 2.5 & 4.8 & 1.5 & 70.9 & 1.2 & 0.5 & 0.7 & 2.9 & 13 & 2 \\
\hline & $\sigma$ & 0.1 & 0.1 & 0 & 0 & 0.1 & 0.1 & 0.1 & 0 & 0.1 & 0 \\
\hline \multirow[t]{2}{*}{7} & BDX16321 & 2.5 & 3 & 1.7 & 75.6 & 1.3 & 0.5 & 0.8 & 3.2 & 9.7 & 1.7 \\
\hline & $\sigma$ & 0 & 0 & 0.1 & 0 & 0 & 0 & 0 & 0 & 0.2 & 0.2 \\
\hline \multirow[t]{2}{*}{8} & BDX16322 & 2.5 & 2.6 & 1.5 & 77 & 1.4 & 0.6 & 0.7 & 3.6 & 8.1 & 2.1 \\
\hline & $\sigma$ & 0.1 & 0.1 & 0 & 0.4 & 0.1 & 0 & 0 & 0 & 0.3 & 0 \\
\hline \multirow[t]{2}{*}{9} & BDX16326 & 2.5 & 4 & 1.6 & 73.2 & 1.1 & 0.5 & 0.7 & 2.7 & 11.8 & 1.9 \\
\hline & $\sigma$ & 0.2 & 0.3 & 0.1 & 0.3 & 0.2 & 0 & 0 & 0.1 & 0.1 & 0.1 \\
\hline \multirow[t]{2}{*}{10} & BDX16327 & 2.5 & 3.9 & 1.9 & 71.7 & 1.2 & 0.4 & 0.6 & 2.6 & 13.3 & 1.8 \\
\hline & $\sigma$ & 0.1 & 0 & 0.1 & 0.6 & 0.2 & 0 & 0.1 & 0.1 & 0.6 & 0.1 \\
\hline \multirow[t]{2}{*}{11} & BDX16328 & 2.4 & 4.5 & 1.5 & 72.1 & 1.2 & 0.5 & 0.9 & 3.1 & 11.9 & 1.9 \\
\hline & $\sigma$ & 0 & 0 & 0 & 0.7 & 0.1 & 0.1 & 0.1 & 0.1 & 0.6 & 0.1 \\
\hline \multirow[t]{2}{*}{12} & BDX16329 & 2.6 & 5.6 & 1.8 & 70.8 & 1.3 & 0.5 & 0.5 & 3 & 11.9 & 2.1 \\
\hline & $\sigma$ & 0 & 0.6 & 0.1 & 1.9 & 0 & 0 & 0 & 0.2 & 0.7 & 0.2 \\
\hline \multirow[t]{2}{*}{13} & BDX16330 & 2 & 4.3 & 1.8 & 73.2 & 1.2 & 0.5 & 0.8 & 2.4 & 11.9 & 1.9 \\
\hline & $\sigma$ & 0.1 & 0.1 & 0.2 & 0.4 & 0.1 & 0 & 0.1 & 0 & 0.1 & 0 \\
\hline \multirow[t]{2}{*}{14} & BDX16331 & 2.6 & 5.3 & 1.6 & 70.5 & 1.3 & 0.5 & 0.7 & 3 & 12.6 & 1.9 \\
\hline & $\sigma$ & 0.1 & 0.1 & 0 & 0.4 & 0.1 & 0 & 0 & 0.1 & 0.3 & 0.1 \\
\hline \multirow[t]{2}{*}{15} & BDX16332 & 3.1 & 3.4 & 1.5 & 68 & 1.4 & 0.7 & 0.9 & 6.2 & 11.4 & 3.3 \\
\hline & $\sigma$ & 0.1 & 0.6 & 0.1 & 0.5 & 0.1 & 0 & 0.1 & 0.1 & 0.1 & 0.2 \\
\hline \multirow[t]{2}{*}{16} & BDX16333 & 2.6 & 4.4 & 1.6 & 71.6 & 1.3 & 0.5 & 0.7 & 2.9 & 12.4 & 2 \\
\hline & $\sigma$ & 0.1 & 0.4 & 0 & 0.8 & 0.2 & 0 & 0.1 & 0.3 & 0.5 & 0.1 \\
\hline \multirow[t]{2}{*}{17} & BDX16342 & 4.3 & 4.8 & 1.3 & 68.3 & 1.7 & 0.7 & 0.9 & 4.8 & 10.1 & 3.1 \\
\hline & $\sigma$ & 0.1 & 0.9 & 0.1 & 1.9 & 0.1 & 0.1 & 0.1 & 0.4 & 0.9 & 0 \\
\hline \multirow[t]{2}{*}{18} & BDX16343 & 2.3 & 5.3 & 1.5 & 74.8 & 1.3 & 0.4 & 0.7 & 2.9 & 9.1 & 1.7 \\
\hline & $\sigma$ & 0.1 & 0.3 & 0.1 & 0.3 & 0.1 & 0 & 0 & 0.2 & 0.2 & 0 \\
\hline \multirow[t]{2}{*}{19} & BDX16345 & 3.3 & 3.1 & 2.3 & 72.2 & 1.4 & 0.6 & 0.9 & 4.6 & 8.9 & 2.7 \\
\hline & $\sigma$ & 0.1 & 0 & 0.9 & 0.9 & 0 & 0 & 0 & 0.1 & 0.1 & 0.1 \\
\hline \multirow[t]{2}{*}{20} & BDX17162 & 2.6 & 3.7 & 1.6 & 74.9 & 1.3 & 0.5 & 0.8 & 3.2 & 9.6 & 1.9 \\
\hline & $\sigma$ & 0.1 & 0.1 & 0 & 0.5 & 0.1 & 0 & 0.1 & 0.2 & 0.1 & 0 \\
\hline 21 & BDX17166 & 2.9 & 2.6 & 1.5 & 73.5 & 1.2 & 0.7 & 0.9 & 4.3 & 9.9 & 2.5 \\
\hline 22 & BDX17167 & 3 & 3.7 & 1.7 & 74.1 & 1.3 & 0.6 & 0.9 & 3.7 & 8.8 & 2.2 \\
\hline & $\sigma$ & 0.1 & 0.3 & 0 & 0.1 & 0.1 & 0.1 & 0 & 0.1 & 0.1 & 0.2 \\
\hline 23 & BDX17168 & 2.5 & 5 & 1.6 & 72 & 1.4 & 0.5 & 0.8 & 3.1 & 11.1 & 1.9 \\
\hline & $\sigma$ & 0.3 & 1.5 & 0.1 & 2.4 & 0.4 & 0.1 & 0.1 & 0.2 & 0.3 & 0.1 \\
\hline 24 & BDX17169 & 2.5 & 4.6 & 1.7 & 71.2 & 1.1 & 0.5 & 0.6 & 2.9 & 13 & 1.9 \\
\hline & $\sigma$ & 0.3 & 0.3 & 0.1 & 0.3 & 0 & 0 & 0 & 0.1 & 0.3 & 0.1 \\
\hline 25 & BDX17170 & 2.7 & 3.4 & 1.7 & 74.8 & 1.2 & 0.6 & 0.7 & 3.7 & 9 & 2.2 \\
\hline & $\sigma$ & 0 & 0.2 & 0.1 & 0.7 & 0 & 0 & 0 & 0.1 & 0.4 & 0 \\
\hline
\end{tabular}


Table 2 continued

\begin{tabular}{|c|c|c|c|c|c|c|c|c|c|c|c|}
\hline & Sample ID & $\mathrm{KKa}_{1}$ & $\mathrm{CaKa}_{1}$ & $\mathrm{MnKa}_{1}$ & $\mathrm{FeKa}_{1}$ & $\mathrm{ZnKa}_{1}$ & $\mathrm{GaKa}_{1}$ & AsKa $_{1}$ & $\mathrm{RbKa}_{1}$ & $\mathrm{SrKa}_{1}$ & $\mathrm{YKa}_{1}$ \\
\hline \multirow[t]{2}{*}{26} & BDX17171 & 2.6 & 2.5 & 1.2 & 79.1 & 1.2 & 0.5 & 0.6 & 3.2 & 6.8 & 2.3 \\
\hline & $\sigma$ & 0 & 0.2 & 0.1 & 0.5 & 0.1 & 0 & 0 & 0 & 0.2 & 0.1 \\
\hline \multirow[t]{2}{*}{27} & BDX17172 & 2.7 & 2.5 & 1.5 & 76.1 & 1.2 & 0.5 & 0.7 & 3.8 & 8.9 & 2.1 \\
\hline & $\sigma$ & 0.1 & 0.2 & 0.1 & 0.2 & 0.1 & 0.1 & 0 & 0.1 & 0.2 & 0.1 \\
\hline \multirow[t]{2}{*}{28} & BDX17173 & 2.7 & 3.2 & 1.1 & 75 & 1.3 & 0.6 & 0.8 & 3.6 & 9.5 & 2.3 \\
\hline & $\sigma$ & 0.1 & 0.5 & 0 & 0.1 & 0 & 0.1 & 0 & 0.2 & 0.5 & 0.2 \\
\hline \multirow[t]{2}{*}{29} & BDX17174 & 2.8 & 2.9 & 1.4 & 73.8 & 1.4 & 0.6 & 0.8 & 3.9 & 10 & 2.5 \\
\hline & $\sigma$ & 0.3 & 0.2 & 0 & 1.3 & 0.1 & 0 & 0 & 0.1 & 0.3 & 0.3 \\
\hline \multirow[t]{2}{*}{30} & BDX17175 & 3.2 & 3.5 & 1.4 & 73.7 & 1.3 & 0.6 & 0.8 & 3.8 & 9.1 & 2.5 \\
\hline & $\sigma$ & 0.1 & 0.2 & 0 & 0.9 & 0.1 & 0 & 0 & 0 & 0.5 & 0 \\
\hline \multirow[t]{2}{*}{31} & BDX17176 & 2.4 & 4.4 & 1.6 & 73.8 & 1 & 0.5 & 0.7 & 2.9 & 10.9 & 1.9 \\
\hline & $\sigma$ & 0.1 & 0.1 & 0.1 & 0.2 & 0.1 & 0 & 0.1 & 0.2 & 0.1 & 0 \\
\hline \multirow[t]{2}{*}{32} & BDX17177 & 3.6 & 4.3 & 1.6 & 70.5 & 1.4 & 0.5 & 0.8 & 3.4 & 11.6 & 2.1 \\
\hline & $\sigma$ & 0.1 & 0.1 & 0.1 & 1.4 & 0.3 & 0 & 0.1 & 0.1 & 0.6 & 0.2 \\
\hline \multirow[t]{2}{*}{33} & BDX17178 & 2.8 & 4.6 & 1.8 & 73.3 & 1.2 & 0.6 & 0.7 & 2.9 & 10.2 & 1.9 \\
\hline & $\sigma$ & 0 & 0.7 & 0 & 0.4 & 0 & 0 & 0 & 0 & 0.3 & 0 \\
\hline \multirow[t]{2}{*}{34} & BDX17179 & 3 & 5.3 & 1.7 & 68.7 & 1.7 & 0.6 & 0.7 & 3.2 & 12.9 & 2.2 \\
\hline & $\sigma$ & 0.3 & 0.1 & 0.1 & 0.2 & 0.2 & 0 & 0.1 & 0.1 & 0.4 & 0.1 \\
\hline \multirow[t]{2}{*}{35} & BDX17180 & 2.5 & 5.5 & 1.4 & 69.7 & 1.3 & 0.6 & 0.8 & 2.7 & 13.5 & 1.9 \\
\hline & $\sigma$ & 0.1 & 0.4 & 0.1 & 0.9 & 0.1 & 0 & 0.1 & 0.1 & 0.4 & 0 \\
\hline \multirow[t]{2}{*}{36} & BDX17181 & 3.2 & 6.1 & 1.6 & 67.3 & 1.5 & 0.6 & 0.9 & 4.4 & 11.6 & 2.8 \\
\hline & $\sigma$ & 0 & 1.3 & 0.1 & 2 & 0.1 & 0.1 & 0.1 & 0.2 & 0.7 & 0 \\
\hline \multirow[t]{2}{*}{37} & BDX17182 & 2.3 & 3.3 & 1.5 & 74.2 & 1.1 & 0.5 & 0.6 & 2.5 & 12.2 & 1.8 \\
\hline & $\sigma$ & 0.2 & 0.1 & 0.1 & 1.5 & 0 & 0.1 & 0.1 & 0.1 & 1.1 & 0.1 \\
\hline \multirow[t]{2}{*}{38} & BDX16334 & 3 & 6.6 & 1.8 & 66.9 & 1.4 & 0.6 & 1.1 & 2.3 & 14.5 & 2 \\
\hline & $\sigma$ & 0.2 & 0.9 & 0 & 1.3 & 0 & 0.1 & 0 & 0.1 & 0.4 & 0 \\
\hline \multirow[t]{2}{*}{39} & BDX16335 & 2.9 & 5.4 & 1.7 & 67.7 & 1.2 & 0.5 & 1 & 2.4 & 15.4 & 1.8 \\
\hline & $\sigma$ & 0 & 0.1 & 0.2 & 0.3 & 0.1 & 0.1 & 0 & 0 & 0.5 & 0.1 \\
\hline \multirow[t]{2}{*}{40} & BDX16339 & 2.8 & 6.5 & 1.7 & 70.8 & 1.3 & 0.6 & 0.7 & 3.1 & 10.4 & 2.1 \\
\hline & $\sigma$ & 0 & 0.2 & 0.1 & 0 & 0.1 & 0.1 & 0 & 0.2 & 0.2 & 0.1 \\
\hline 41 & BDX16340 & 2.7 & 5.3 & 1.6 & 72.7 & 1.4 & 0.4 & 0.6 & 3.2 & 10.2 & 2.1 \\
\hline 42 & BDX17164 & 2.8 & 4.5 & 1.6 & 71.7 & 1.2 & 0.5 & 0.8 & 3.3 & 11.3 & 2.2 \\
\hline 43 & BDX17165 & 2.5 & 5.9 & 1.7 & 72.3 & 1.1 & 0.5 & 0.6 & 3.2 & 10.5 & 1.7 \\
\hline \multirow[t]{2}{*}{44} & BDX16553 & 2 & 1.8 & 1.7 & 81.5 & 1.2 & 0.5 & 0.5 & 1.5 & 8.3 & 1.1 \\
\hline & $\sigma$ & 0.1 & 0.1 & 0.1 & 0.7 & 0.1 & 0 & 0 & 0 & 0.4 & 0 \\
\hline \multirow[t]{2}{*}{45} & BDX16554 & 2.1 & 2.2 & 1.6 & 82.3 & 1.1 & 0.5 & 0.6 & 1.8 & 6.3 & 1.6 \\
\hline & $\sigma$ & 0.1 & 0 & 0.1 & 0.4 & 0.1 & 0.1 & 0 & 0.1 & 0.3 & 0 \\
\hline \multirow[t]{2}{*}{46} & BDX16555 & 1.7 & 1.9 & 2.3 & 81.3 & 1 & 0.4 & 0.4 & 1.2 & 8.6 & 1.2 \\
\hline & $\sigma$ & 0.2 & 0.3 & 1 & 1 & 0.1 & 0 & 0 & 0.1 & 1.2 & 0 \\
\hline 47 & BDX16556 & 1.9 & 2.5 & 1.5 & 80.6 & 1.3 & 0.5 & 0.4 & 1.3 & 8.6 & 1.3 \\
\hline & $\sigma$ & 0.3 & 0.2 & 0.3 & 0.1 & 0.2 & 0 & 0 & 0 & 0.2 & 0 \\
\hline 48 & BDX16557 & 2.8 & 2.5 & 1.5 & 75.8 & 1.1 & 0.5 & 0.5 & 1.3 & 12.7 & 1.3 \\
\hline & $\sigma$ & 0.5 & 0.2 & 0.1 & 1.6 & 0.1 & 0 & 0.1 & 0 & 0.5 & 0.1 \\
\hline 49 & BDX16558 & 1.8 & 2.2 & 2.7 & 79.3 & 0.9 & 0.5 & 0.5 & 1.3 & 9.6 & 1.3 \\
\hline & $\sigma$ & 0.1 & 0.1 & 0.1 & 0.5 & 0 & 0.1 & 0 & 0.1 & 0.2 & 0.1 \\
\hline 50 & BDX16561 & 2.1 & 2.5 & 1.7 & 78.9 & 1.1 & 0.5 & 0.4 & 1.3 & 10 & 1.4 \\
\hline & $\sigma$ & 0.1 & 0.1 & 0.1 & 0.3 & 0.1 & 0 & 0.1 & 0.1 & 0.1 & 0.1 \\
\hline 51 & BDX16559 & 4.3 & 3.1 & 1.5 & 48.5 & 1.8 & 1 & 1.1 & 7.7 & 24.5 & 6.6 \\
\hline & $\sigma$ & 0.9 & 0.4 & 0.2 & 1.9 & 0.1 & 0.2 & 0.1 & 0.4 & 0.2 & 0.7 \\
\hline
\end{tabular}


Table 2 continued

\begin{tabular}{|c|c|c|c|c|c|c|c|c|c|c|c|}
\hline & Sample ID & $\mathrm{KKa}_{1}$ & $\mathrm{CaKa}_{1}$ & $\mathrm{MnKa}_{1}$ & $\mathrm{FeKa}_{1}$ & $\mathrm{ZnKa}_{1}$ & $\mathrm{GaKa}_{1}$ & $\mathrm{AsKa}_{1}$ & $\mathrm{RbKa}_{1}$ & $\mathrm{SrKa}_{1}$ & $\mathrm{YKa}_{1}$ \\
\hline 52 & BDX16563 & 3.2 & 2.8 & 1.4 & 56.3 & 1.4 & 0.9 & 1 & 4 & 25.7 & 3.2 \\
\hline \multirow[t]{2}{*}{53} & BDX16464 & 3.3 & 2.7 & 1.2 & 47.5 & 1.5 & 0.8 & 0.9 & 4.2 & 34.8 & 3.1 \\
\hline & $\sigma$ & 0.6 & 0.2 & 0.1 & 3.5 & 0.3 & 0.1 & 0.2 & 0.4 & 1.7 & 0.2 \\
\hline \multirow[t]{2}{*}{54} & BDX16565 & 4.3 & 4.2 & 1.3 & 57.3 & 1.7 & 1.1 & 0.9 & 5 & 20.5 & 3.7 \\
\hline & $\sigma$ & 0.1 & 0.3 & 0.2 & 0.6 & 0 & 0.1 & 0.1 & 0.4 & 0.1 & 0.1 \\
\hline \multirow[t]{2}{*}{55} & BDX16566 & 3.6 & 2.9 & 1.7 & 55.7 & 1.5 & 0.9 & 0.9 & 7 & 20.5 & 5.4 \\
\hline & $\sigma$ & 0.1 & 0.3 & 0.1 & 1.4 & 0.1 & 0 & 0.2 & 0.1 & 1.2 & 0.5 \\
\hline \multirow[t]{2}{*}{56} & BDX16567 & 3.9 & 4.8 & 1.4 & 52.7 & 1.5 & 0.9 & 0.9 & 6.7 & 21.7 & 5.4 \\
\hline & $\sigma$ & 0.1 & 0.2 & 0.2 & 0.2 & 0.2 & 0.2 & 0.2 & 0.2 & 0.2 & 0.2 \\
\hline
\end{tabular}
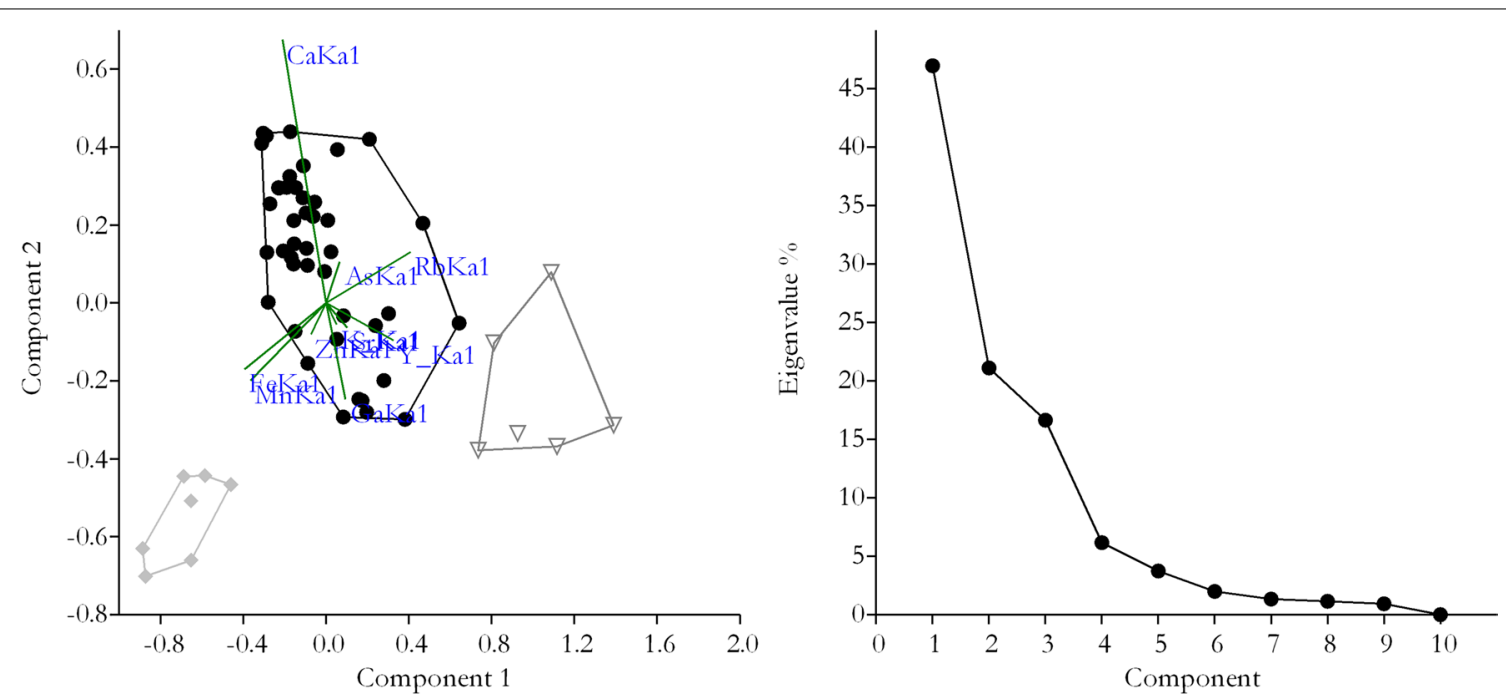

Fig. 3 PCA biplot (left) and PCA scree plot (right) using CLR transformed HHpXRF raw intensities data: Mochica bichrome and Mochica polychrome ceramics (black dots), Coastal Cajamarca (gray diamonds) and Highland Cajamarca ceramics (gray triangles). Var-cov matrix was employed to project all compositional information. Convex hulls were denoted

While in the SJM-2 fragments, $\mathrm{SrK} \alpha_{1} / \mathrm{FeK}_{1}$ ratio was $0.08-0.17$, and $\mathrm{RbK} \alpha_{1} / \mathrm{SrK} \alpha_{1}$ ratio was $0.38-0.54$.

In order to compare all the Mochica fragments to ratify and confirm the presence of these two chemical subgroups; a fully cross-validated PCA analysis considers all the chemical elements. The analysis of raw intensities data transformed (CLR transformation) inside this group (notably for $\mathrm{CaK} \alpha_{1}, \mathrm{SrK \alpha}_{1}, \mathrm{RbK} \alpha_{1}$ and $\mathrm{YK} \alpha_{1}$ data) demonstrates the existence of two chemical sub-groups that are not directly related to the bichrome or polychrome ceramics (Fig. 4).

The chemical sub-group SJM-1 is represented by the black dots and the chemical sub-group SJM-2 is represented by the gray triangles. The sub-group SJM-1 consists of 30 sherds: 24 sherds of Mochica fineline bichrome and notably all of the 6 sherds of the Mochica fineline polychrome. In contrast SJM-2 group also included 12 fragments of Mochica fineline bichrome. A single sample (BDX16321) could not directly assimilate to one of two sub-groups by where $\mathrm{SrK} \alpha_{1} / \mathrm{FeK} \alpha_{1}$ ratio of 0.13 and $\mathrm{RbK} \alpha_{1} / \mathrm{SrK \alpha}_{1}$ ratio of 0.33 lies at the interface of SJM-1 and SJM-2.

Notably in SJM-1, the Mochica bichrome painted samples and Mochica polychrome painted samples overlap and are indistinguishable. Both styles have $\mathrm{Ca}$ rich ceramic bodies quite likely produced in local workshops using mainly the same local raw materials [34], and elaborated without the same recipe used in the Coastal and Highland Cajamarca productions. The SJM-2 group is both aesthetically and chemically different from the Cajamarca ceramics. Currently, there lacks the data needed to distinguish whether this Mochica sub-group (SJM-2) was or not produced locally by San José de Moro potters. 

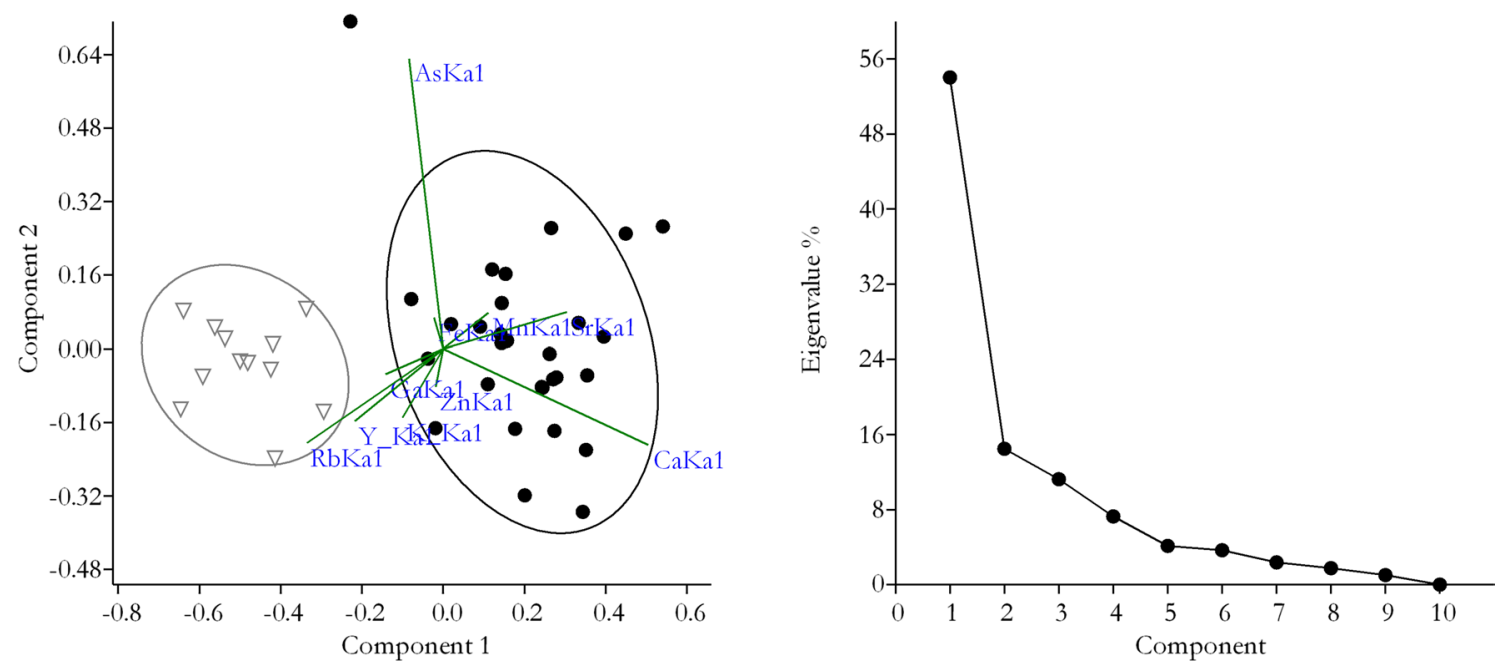

Fig. 4 PCA biplot (left) and PCA scree plot (right) using CLR transformed HHpXRF raw intensities data. "SJM-1": black dots (85\% confidence ellipse); "SJM-2": gray triangles (85\% confidence ellipse). Var-cov matrix was employed to project all compositional information

\section{Conclusions}

Each fragment was macroscopically investigated, identified and grouped. Following a nondestructive archaeometric approach, we engaged the use of HHpXRF spectrometry and performed chemical characterization analysis on all samples. Our results strongly support the aesthetic approach documented by archaeologists coupled with the HHpXRF technique in classifying all samples into the same three groups-Mochica, Coastal Cajamarca and Highland Cajamarca.

Thus, in situ HHpXRF analysis further established that sherds identified as aesthetically different also differ chemically due to the use and combination of distinctive raw materials, with the exception of Mochica fineline painted styles. Even while Mochica and Cajamarca people were increasing the trade of artefacts and breaking the status-quo of cultural interaction, potters of both societies did not exchange raw materials: Coastal and Highland Cajamarca potters did not produced ceramic bodies using the raw materials exploited by Mochica potters. Mochica, Coastal Cajamarca and Highland Cajamarca ceramic of these periods are aesthetically and chemically different.

Lastly, the specific analysis of Mochica bichrome and polychrome fragments showed the existence of two chemical sub-groups: SJM-1 and SJM-2. Both are not surprisingly related to the decoration and color aspects of objects. SJM-1 is the sub-group the most representative of the SJM site. In addition, if the Mochica fineline bichrome ceramic production could be classified as homogenous, Mochica fineline polychrome ceramic production was indeed standardized. All Mochica fineline polychrome ceramics were produce locally as well.

With the correct research questions, a good methodology and an understanding of its parameters, HHpXRF is a valuable aid to an archaeologist's on-site thought process. New methodologies in HHpXRF would open the way to formulate and test new theories regarding cultural, behavioural and intellectual aspects of our recent ancestors. HHpXRF theoretical approaches leaning toward space, context and material patterning will open new ways of collecting data in situ.

\section{Authors' contributions}

NDS carried out the laboratory experiments. RC, LC, RJB provided support and guidance for this study. NDS, SK, RC, LC and RJB analyzed the data, interpreted the results and drafted the paper. All authors have contributed to, seen and approved the manuscript. All authors read and approved the final manuscript.

\section{Author details}

${ }^{1}$ Institut de Recherche sur les Archéomatériaux-Centre de Recherche en Physique Appliquée à l'Archéologie (IRAMAT-CRP2A) UMR 5060 CNRS, Université Bordeaux Montaigne, Bordeaux, France. ${ }^{2}$ Southern Cross GeoScience, Southern Cross University, Lismore, Australia. ${ }^{3}$ Proyecto Arqueológico San José de Moro (PASJM), Pontificia Universidad Católica del Perú, Lima, Peru.

\section{Acknowledgements}

We thank the IdEx program of the University of Bordeaux (France) and the cluster of excellence called "laboratoire des sciences archéologiques de Bordeaux: LaScArBx" (ANR-10-LABX-52).

\section{Competing interests}

The authors declare that they have no competing interests.

Received: 9 June 2016 Accepted: 15 October 2016

Published online: 17 November 2016 


\section{References}

1. Castillo LJ. La presencia Wari en San José de Moro. In: Makowski K, editor. Los dioses del antiguo Perú. Lima: Colección Arte y Tesoros del Perú; 2000. p. 103-35

2. Castillo LJ. Los últimos mochicas en Jequetepeque. In: Uceda S, Mujica E, editors. Moche: hacia el final del milenio: Actas del segundo coloquio sobre la cultura moche (1-7 agosto 1999). Lima: Pontificia Universidad Católica del Perú \& Universidad Nacional de Trujillo; 2003. p. 65-123.

3. Castillo LJ. Prácticas funerarias de élite en San José de Moro. In: Makowski K, editor. Los Señores de los reinos de la luna. Lima: Banco de Crédito del Perú; 2008. p. 288-93.

4. Donnan C, McClelland D. Moche fineline painting, its evolution and its artist. Los Angeles: UCLA Fowler Museum; 1999.

5. McClelland D, Mcclelland D, Donnan C. Moche fineline painting from San José de Moro. Los Angeles: The Cotsen Institute of Archaeology Press; 2007.

6. Castillo L, Uceda S. The Mochicas. In: Silverman H, Isbell W, editors. Handbook of South American Archaeology. Hoboken: Blackwell Press; 2008. p. 707-29.

7. Rohfritsch A. Céramiques mochicas de la vallée de Jequetepeque (Pérou): étude technique et physicochimique d'exemplaires provenant de Dos Cabezas et San José de Moro. Mémoire Master 2, Université Michel de Montaigne Bordeaux 3; 2006.

8. Thiriet C. Les céramiques Cajamarca du site de San José de Moro (Pérou): étude comparative des groupes stylistiques « serrano et costeño». Mémoire Master 2, Université Michel de Montaigne Bordeaux 3; 2008.

9. Del Solar N. La caractérisation archéométrique des céramiques précolombiennes du Pérou: une synthèse historique et méthodologique. Mémoire Master 1, Université Michel de Montaigne Bordeaux 3; 2011.

10. Saldaña J, Tavera A, Patroni K. Informe técnico de las excavaciones en el área 47 de San José de Moro. Report of the Programa Arqueológico de San José de Moro Temporada 2013. Lima: Pontificia Universidad Católica del Perú; 2013.

11. Castillo LJ. San José de Moro y el Fin de los Mochicas en el Valle de Jequetepeque, Costa Norte del Peru. Ph.D. dissertation, University of California; 2012

12. Herrera M, Clauwaerts P. Informe técnico de las excavaciones en el área 49 de San José de Moro Report of the Programa Arqueológico de San José de Moro Temporada 2013. Lima: Pontificia Universidad Católica del Perú; 2013.

13. Palomino R, Miller M. Informe técnico de las excavaciones en el área 50 de San José de Moro Report of the Programa Arqueológico de San José de Moro Temporada 2013. Lima: Pontificia Universidad Católica del Perú 2013

14. Rohfritsch A. Contribución arqueométrica al estudio de las técnicas y de la organización de la producción de cerámica ritual en la sociedad Mochica (150-850 d.C., costa norte del Perú). Bull Inst fr étud andin. 2010;39(2):389-412

15. Druc I, Inokuchi K, Shen Z. Análisis de arcillas y material comparativo por medio de Difracción de rayos $X$ y Petrografía para Kuntur Wasi, Cajamarca. Perú Arqueol Soc. 2013;26:91-110.

16. Koons M. External versus internal: an examination of moche politics through similarities and differences in ceramic style. In: Druc I, editor. Ceramic Analysis in the Andes. Wisconsin: Deep University Press; 2015. p. 57-82.
17. Pena JL. Pottery production during the late horizon in the Huancabamba Valley, Cajamarca-Peru. Master of Arts thesis, University of South Florida; 2013.

18. Frahm E, Doonan R. The technological versus methodological revolution of portable XRF in archaeology (review). Archaeol Sci. 2013;40(2):142534. doi:10.1016/j.jas.2012.10.013.

19. Speakman R, Shackley M. Silo science and portable XRF in archaeology a response to Frahm. [j Archaeol Sci. 2013;40:1435-43.

20. Killick D. The awkward adolescence of archaeological science. J Archaeol Sci. 2015;56:242-7.

21. Clarke D. Archaeology: the loss of innocence. Antiquity. 1973;47(185):6-18.

22. Hodder I. Reading the past. Cambridge: Cambridge University Press; 1986.

23. Shanks M, Tilley C. Re-constructing archaeology: theory and practice. Cambridge: Cambridge University Press; 1987.

24. Andrews K, Doonan R. Test tubes \& trowels: using science in archaeology. Stroud:Tempus; 2003

25. Speakman R, Little N, Creel D, Miller M, Iñañez J. Sourcing ceramics with portable XRF spectrometers? A comparison with INAA using Mimbres pottery from the American Southwest. J Archaeol Sci. 2011;38(12):3483-96.

26. Aitchison J. The statistical analysis of compositional data-monographs on statistics and applied probability. London: Springer; 1986.

27. Buxeda I, Garrigós J. Alteration and contamination of archaeological ceramics: the perturbation problem. J Archaeol Sci. 1999;26:295-313.

28. Machut P, Ben Amara A, Cantin N, Chapoulie R, Frèrebeau N, Le Bourdonnec FX. Towards high resolution ceramic series for production site studies: the case of Loron amphorae (Croatia 1st-3rd c. A.D.). Heritage Sci. 2015;3:1. doi:10.1186/s40494-015-0050-5.

29. Hammer $\varnothing$, Harper $D$, Ryan P. PAST: paleontological statistics software package for education and data analysis (v2.17). Palaeontol Electron. 2001;4(1):9.

30. Bernuy K, Bernal V. La tradición Cajamarca en San José de Moro: una evidencia de interacción interregional durante el Horizonte Medio. In: Castillo LJ, Bernier H, Lockard G, Rucabado J, editors. Arqueología mochica: nuevos enfoques: Actas del primer Congreso internacional de jóvenes investigadores de la cultura mochica (4-5 agosto 2004). Lima: Pontificia Universidad Católica del Perú \& Institute Français des Études Andines; 2008. p. 67-80.

31. Terada K, Matsumoto K. Sobre la cronología de la tradición Cajamarca. Historia de Cajamarca: Arqueología. 1985:1:67-89.

32. Watanabe S. Wari y Cajamarca. Bol Arqueol PUCP. 2001;5:531-41.

33. La Watanabe S. Cerámica caolín en la cultura Cajamarca (sierra norte del Perú): el caso de la fase Cajamarca Media. Bull Inst fr étud andin. 2009;38(2):205-36.

34. Del Solar N. Contribution des sciences archéologiques à la connaissance des choix techniques Mochica et Cajamarca: Étude des matériaux céramiques du site de San José de Moro (VIII ${ }^{\mathrm{e}} \mathrm{X}^{\mathrm{e}}$ s. apr. J.-C., Pérou). Ph.D. dissertation, Université Bordeaux Montaigne; 2015.

\section{Submit your manuscript to a SpringerOpen ${ }^{\oplus}$ journal and benefit from:}

- Convenient online submission

- Rigorous peer review

- Immediate publication on acceptance

- Open access: articles freely available online

- High visibility within the field

- Retaining the copyright to your article

Submit your next manuscript at $>$ springeropen.com 